In press at Current Opinion in Psychology.

This version of the manuscript is not the copy of record and may not exactly represent the

final, authoritative version of the article.

Helping in the context of refugee immigration

\author{
Gerald Echterhoff ${ }^{\mathrm{a}}$ \\ Julia C. Becker ${ }^{b}$ \\ Judith Knausenberger ${ }^{\mathrm{a}}$ \\ Jens H. Hellmann ${ }^{\mathrm{a}}$
}
${ }^{a}$ University of Münster, Institute of Psychology, Fliednerstr. 21, 48149 Münster, Germany
${ }^{\mathrm{b}}$ University of Osnabrück, Department of Psychology, Seminarstr. 20, 49074 Osnabrück, Germany

Gerald Echterhoff g.echterhoff@wwu.de (corresponding author)

Julia C. Becker julia.becker@uni-osnabrueck.de

Judith Knausenberger judith.knausenberger@uni-muenster.de

Jens H. Hellmann jens.hellmann@uni-muenster.de

\title{
Highlights
}

- We focus on psychological approaches of help for refugees

- Based on the PARI model, we highlight the role of migration forcedness and perils

- We consider the four-stage model and different motives of helping

- We discuss factors and consequences of autonomy- vs. dependency-oriented help

- Helping is placed in the context of intergroup power relations 


\begin{abstract}
We review psychological approaches of helping behavior in the context of refugee immigration. Refugee migration, compared to non-refugee migration, is characterized by greater forcedness and related perils. Taking into account perceptions of forcedness and perils, we examine potential helpers' responses at each of four successive stages towards helping people in perilous, distressing, or emergency situations: (1) noticing and recognizing distressing, help-demanding conditions, (2) taking responsibility, (3) knowing how to help, and (4) transfer of one's knowledge into action. In so doing, we discuss the role of different motives and functions of providing help (e.g., preserving refugees' dependency or facilitating their autonomy), and implications of unequal power relations between help providers and refugees.
\end{abstract}

[112 words]

Keywords: helping, refugees, activism, forcedness, perils, autonomy- vs. dependency-oriented help, immigration 
Unprecedentedly high numbers of refugees, reaching approximately 30 million in 2020 [1], represent one of the global crises of the $21^{\text {st }}$ century. A distinctive characteristic of refugees is that they are forced to migrate due to severe push factors in their countries of residence [2]. Forced emigration typically entails a precipitous departure and little opportunity for planning the journey, which increases the likelihood of refugees experiencing risks and life-threatening perils during migration [3]. In addition to these perils of migration, refugees also face adverse and challenging conditions after their arrival in a receiving country, including language barriers, unfamiliar immigration procedures, restricted freedom of movement, confrontation with foreign laws, cultural norms and habits, loss of social status, and discrimination $[4,5]$.

These migration and post-migration conditions put refugees in the position of people who require support, assistance, and help (for legal-ethical arguments, see [6]). In this review, we primarily examine recent research that has examined social-psychological, person and context factors of helping intentions and helping behaviors towards refugees. We focus on helping as intentional action yielding benefits for another person [7,8]. Also, we focus on individuals and groups as potential providers of help rather than on organizations or institutions.

To structure our review, we distinguish between successive stages leading to provision of help for people in perilous, distressing, or emergency situations [9,10]: (i) noticing and recognizing distressing, help-demanding conditions, (ii) taking responsibility, (iii) knowing how to help, and (iv) taking action. Below, we outline factors of potential helpers' responses at each of these stages. Because the findings on noticing and recognizing overlap, we subsume both in one stage.

Wherever pertinent, we refer to distinct features of refugee migration, that is, forcedness of migration and associated perils [3]. We argue that help-promoting responses are 
more likely when forcedness and related perils of migration are more salient and thus more easily perceived.

\subsection{Noticing the situation and recognizing its emergency: How attention is drawn to the} refugees' plight

The characteristics of global crises such as the so-called refugee crisis can impede recognition of the crisis as a current emergency requiring help. These characteristics are (i) low identifiability of victims, (ii) the lack of specific and salient events signaling the emergency, and (iii) the long-term persistency of adverse conditions during extended crises, which can lead to the perception that the situation is a regrettable chronic state rather than an emergency requiring immediate action [11]. Amidst the load of incessantly transmitted media information, residents are more likely to become aware of the perilous conditions of refugee migration when they are confronted with vivid information about individual refugees' suffering.

The impact of victims' identifiability on people's awareness of refugees' plight and willingness to help is epitomized by the tragic death of Aylan Kurdi. The picture of the young boy's body on the beach reached at least 20 million people within 12 hours [12]. In the aftermath, donations to the refugee program of the Swedish Red Cross increased dramatically [13] and tweeting about Aylan Kurdi's death predicted solidarity with refugees ten weeks later [14]. Also, pictures of smaller (vs. larger) refugee groups may increase pity for the depicted refugees [15].

The recognition of the circumstances of refugee migration as an emergency is associated with empathic concern [11]. Research suggests several predictors of empathic concern. First, a study in Italy found that lack of empathic concern is associated with person factors, specifically, residents' prejudice against immigrants, Social Dominance Orientation, Right-Wing Authoritarianism [16]. A second predictor is interpersonal contact, which arguably makes forcedness and perils of refugee migration more salient. More contact with 
refugees is associated with more pity [17], and direct, indirect, and imagined forms of contact are associated with more empathy and helping intentions $[18,19]$.

A third predictor is directly related to perceived forcedness of migration, a distinctive feature of refugee migration [3]. Studies conducted in the Netherlands [20,21] show that residents' empathic concern is enhanced by the perception that migration was involuntary (vs. voluntary), that is, more forced. Learning about a refugee child's perils before and during migration also increases empathic concern and helping intentions in children [22].

A fourth predictor are verbal labels employed for forced migrants. The label "war refugees" elicits more pity than does the label refugees, and both labels are associated with more pity than the label asylum seekers [23] (for labeling effects, see also [24]). "War refugees" explicitly conveys severe push factors experienced by this group of refugees before migration, thus invoking forcedness and perils. Perceptions of greater forcedness and perils of migration are likely to increase the probability of recognizing and interpreting the refugees' situation as an emergency, which predicts helping intentions and supporting charitable organizations [11].

\subsection{Assuming responsibility for helping refugees}

Once conditions of refugees are recognized as help-demanding, the next step for potential help providers is to feel responsible for taking action. At general level, for largescale, widely reported macro-level emergencies such as the "refugee crisis", a common perception is that of numerous other potential helpers, including organizations and institutions, exacerbating diffusion of responsibility [25].

Direct empirical investigations of this stage are scarce. In one study conducted in the UK and Germany, respondents who who felt more similar to Syrian refugees and those who took the first step towards helping (noticing and recognizing help-demanding conditions) were more likely to feel responsible for providing help [11]. 
Furthermore, an analysis of discourse in the media and politics in the UK suggests a differentiated view of the public sense of responsibility [26]. On the one hand, media discourse and politicians generally acknowledge a common ethics of helping innocent victims (i.e., refugees). On the other hand, responsibility for helping is portrayed as requiring refugees' willingness, efforts, and ability to integrate into or assimilate to the majority culture. According to the underlying, hegemonic notion, the majority society shall provide resources but the subordinate minority group is expected to follow the majority's rules. By implication, the majority is responsible for help only when refugees are willing to assimilate.

\subsection{Knowing how to help refugees}

Regarding the next stage, the implementation of helping requires that potential providers know which means and resources are needed for support. Regarding refugees, residents can choose among various types of help. A widely known opportunity are donations to international charitable organizations (e.g., Red Cross), which offer easy, low-threshold procedures for donating. However, this common knowledge can be overshadowed by doubts about the reliable use of funds, exemplified by suspicion of structural inefficiency or corruption of intermediary governmental agencies [25].

Effective help requires knowledge about refugees' needs, contact points, trustworthy institutions, and teaching strategies. According to the PARI model [3], understanding refugees' needs (e.g., for regaining agency, or overcoming distress, anxiety and trauma) is closely associated with the recognition of forcedness and perils of refugee migration. The achievement of this knowledge stands to profit from including representatives of immigrant and refugee communities, as demonstrated by scientific analyses of support programs for refugees [27,28]. The Natural Helpers Program in Dayton, Ohio [28] has yielded a comprehensive overview of preconditions for effective help, such as understanding how to address refugees' social needs (networking, mentoring, communication), economic/material needs (information on government assistance, health insurance, mobility), and emotional 
needs (coping with anxiety, uncertainty, and trauma). Interviews with refugees and resident helpers participating in the Refugee Mentoring program in Melbourne (Australia) revealed that helpers initially faced substantial uncertainties and misunderstandings, which were tentatively diminished by transformational intercultural learning [27].

We note that effective help for refugees suffering from the experience of severe perils (e.g., distress, anxiety, and trauma) requires specific professional knowledge. Such knowledge encompasses diagnostics and treatment of refugees, including relevant culture-specific differences and language issues and therapists' readiness to conduct psychotherapy with refugees [29-31]. Additionally, to seek appropriate help, refugees also need to have relevant knowledge, motivation, and trust in potential mental-health support [32,33].

\subsection{Acting: Providing help to refugees}

When and how do people take action to help refugees? A range of helping behaviors has been suggested including mundane, single acts of helping [32], solidarity, donations, interpersonal help and activism [24,35-41]. Recently, researchers distinguished between two types of helping behavior: benevolence (compassionately alleviating the suffering of others) and activism (changing current social, legal and political structures to create greater equality [42]). These two behaviors can co-occur, but often the actions are driven by different goals and philosophies [41]. Actors motivated by benevolence often help individual victims without addressing the root causes responsible for injustice. In contrast, activists aim to change the system to achieve social change in the future [42]. Thus, the specific motives of people engaging in either form of help needs to be considered to understand the goals of the behavior.

Helping behavior occurs in a context of power inequality. Certain forms of help are used to achieve dominance over lower status groups in order to maintain power hierarchies, whereas other forms of behaviors aim to achieve social equality $[43,44]$. Specifically, researchers distinguished whether a helping behavior is autonomy-oriented or dependency- 
oriented. Autonomy-oriented help enables help seekers to solve the problem on their own after gaining the required skills $[45,46]$. In contrast, dependency-oriented help provides an immediate, full solution of a problem and thus keeps help-recipients in a state of neediness.

When people engage in dependency-oriented (compared to autonomy-oriented) help towards refugees, they are more likely to be paternalistic and have moral image concerns [45], feel sympathy ([37], also see [47]), and view help-recipients as incompetent [48]. In contrast, autonomy-oriented help towards refugees is more likely when individuals experience moral outrage [37], have a group consciousness (shared emotions, group efficacy and identification as refugee supporter [38]), and view refugees as competent [45]. Moral outrage is arguably associated with the recognition of forcedness and perils of refugee migration. Refugees and residents agree that autonomy-oriented help has more potential for social change than dependency-oriented help [45].

Similarly, not all forms of activism seem to benefit the disadvantaged groups. For instance, allyship, that is, support for a target group of injustice (e.g., refugees) among advantaged group members (residents [49]) can be driven by four different motives [50,51]: (i) a genuine interest to improve the status of the disadvantaged group (outgroup-focused motivation), (ii) to help the disadvantaged group as long as their own group does not lose privileges (ingroup-focused motivation), (iii) to meet their own personal needs (personal motivation), and (iv) because this behavior aligns with their personal moral beliefs (morality motivation). Regarding the distinctive factors of refugee migration, the first, outgroupfocused motive should be enhanced by perceptions of migration perils.

However, although some forms of helping behavior seem to be more beneficial for the help recipient than others, the specific situation of refugees and the timing needs to be considered. For instance, directly after refugees entered a new country, basic human needs need to be satisfied (e.g., food, shelter, housing, clothes, medical care). Therefore, immediately after arrival, refugees might need more benevolent forms of helping behavior. 
When the basic human needs are satisfied, months after the arrival, refugees might be more interested in receiving autonomy-oriented help and might appreciate residents' activism for more refugee rights or against deportation [52]. Thus, rather than suggesting that some helping behaviors are more helpful than others, it is important to consider the specific situation of refugees, the timing, and to directly ask refugees what kind of support they need.

\section{Discussion and outlook}

Our review has focused on factors that affect residents' helping behaviors towards refugees after arrival in a receiving society, highlighting the role of residents' recognition of refugees' forcedness and perils of migration. We have argued that this recognition tends to enhance the likelihood of residents' helping responses and helping behavior. However, we note that these perceptions may also induce anxiety and aversion among residents who are more likely to perceive the arrival of strangers from different cultures as threatening [3], which is at odds with refugee helping.

This being said, research is needed to pinpoint the impact of perceived forcedness and perils of migration on all stages towards helping, particularly potential helpers' sense of personal responsibility. For instance, it is possible that the recognition of migrants' forcedness and perils contributes to feelings of responsibility.

As we have emphasized, helping occurs in a context of unequal power relations and can be strategically used to maintain group-based social hierarchies. To mitigate this issue, help recipients need to be involved regarding the kind of help they need (for examples, see $[27,28])$. Some teaching and learning guides have been developed for intergroup prosociality, which could be beneficial for volunteers working with refugees [53].

Clearly, refugees also need help during the migration journey, for instance, due to distressing and perilous conditions of crossing borders, hazardous sea or desert passages, and interim confinement. However, psychological factors of help at this stage of migration are underexamined (for case studies on mutual help among refugees, see [54, 55]). 
Related to this issue, the reviewed literature suggests that autonomy-oriented help can empower refugees to achieve competencies that gradually make external help dispensable. Once refugees overcome experienced loss of control and agency from migration forcedness and regain a sufficient sense of self-efficacy, they are in the position to act as help providers. There are several organizations in which refugees provide help for newly arrived refugees. For instance, the initiative Refugees helping Refugees aims to foster the growth, selfdetermination, and self-reliance of refugees by "making them agents of their own advancement" (https://rhrroc.org/). They offer, for instance, English Language classes, case management, healthy living programs, vocational training, senior services and legal counseling. Help providers who have been former refugees are considered as bridge-builders between newcomers and others in the community [56]. These help providers often feel obligated to help newcomers and a sense of duty, or they perceive their ethnic community as an extension of family [57]. However, psychological research on refugees as help providers is rare. It would be an interesting avenue for future work to focus on the question how refugees turn from recipients to providers of help.

To conclude, scarce but growing research suggests several psychological factors of helping behavior towards refugees. Perceptions of migration forcedness and related perils can moderate effects of these factors at various stages that precede actual helping. Perceptions of threatening or distressing circumstances, especially from vivid individual cases, can increase empathic concern and awareness of the need for help. Also, the knowledge of how to help profits from liaising with refugee communities and diversity-sensitive learning about specific stressors and needs of refugees. Finally, perceptions of refugees' migration forcedness and related perils can boost the likelihood of taking action by promoting genuine interest in refugees' wellbeing. 


\section{References and Recommended Reading}

1. United Nations High Commissioner for Refugees: UNHCR global trends: Forced displacement in 2020. https://www.unhcr.org/statistics/unhcrstats/60b638e37/globaltrends-forced-displacement-2020.html; 2021.

2. Richmond AH: Reactive migration: Sociological perspectives on refugee movements. $J$ Refugee Studies 1993, 6(1):7-24, https://doi.org/10.1093/jrs/6.1.7.

3. **Echterhoff G, Hellmann JH, Back MD, Kärtner J, Morina N, Hertel G: Psychological antecedents of refugee integration (PARI). Perspect Psychol Sci 2020, 15(4):856879. https://doi.org/10.1177/1745691619898838.

In their model of Psychological Antecedents of Refugee Integration (PARI), the authors identify migration forcedness and associated perils as distinctive features of refugee migration, which moderate refugees' and residents' responses to the demands and stressors of immigration. On implication is that residents' helping behavior towards migrants depends on their perceptions and recognition of migration forcedness and associated perils.

4. Kuo BCH: Coping, acculturation, and psychological adaptation among migrants: A theoretical and empirical review and synthesis of the literature. Health Psych and Behav Med 2014, 2(1):16-33. https://doi.org/10.1080/21642850.2013.843459.

5. Stuart J, Nowosad, J: The influence of premigration trauma exposure and early postmigration stressors on changes in mental health over time among refugees in Australia. J Traumatic Stress 2020, 33.6:917-927. https://doi.org/10.1002/jts.22586.

6. Tiedemann P: Are there moral duties toward refugees? Considerations in legal ethics. Laws 2021, 10(1):4. https://doi.org/10.3390/laws10010004.

7. Dovidio JF, Penner, LA: Helping and altruism. In Brewer MB, Hewstone M (Eds.), Emotion and motivation (pp. 247-280). Blackwell Publishing; 2004 
8. Eisenberg N, Miller PA: The relation of empathy to prosocial and related behaviors. Psych Bull 1987, 101(1):91-119. https://doi.org/10.1037/0033-2909.101.1.91

9. Latané B, Darley, JM: The unresponsive bystander: Why doesn't he help? AppletonCentury-Crofts; 1970.

10. Banyard VL: Who will help prevent sexual violence: Creating an ecological model of bystander intervention. Psychol of Violence 2011, 1:216-229. https://doi.org/10.1037/a002373

11. **Albayrak-Aydemir N, Gleibs IH: Measuring global bystander intervention and exploring its antecedents for helping refugees. Br J Psychol 2021, 112(2):519-548. https://doi.org/10.1111/bjop.12474.

This paper is the first to apply the classical bystander effect to the refugee crisis. Two studies validated a scale to measure global bystander intervention for helping refugees. A third study explored how the different stages of the bystander intervention predict subsequent stages and examined antecedents of the different stages in a cross-cultural comparison (UK vs. Germany).

12. Vis F, Goriunova, O: The iconic image on social media: A rapid research response to the death of Aylan Kurdi. Visual Social Media Lab 2015.

13. Slovic P, Västfjäll D, Erlandsson A, Gregory R: Iconic photographs and the ebb and flow of empathic response to humanitarian disasters. PNAS 2017, 114(4):640-644. https://doi.org/10.1073/pnas.1613977114.

14. Smith LG, McGarty C, Thomas EF: After Aylan Kurdi: How tweeting about death, threat, and harm predict increased expressions of solidarity with refugees over time. Psychol Sci 2018, 29(4):623-634. https://doi.org/10.1177/0956797617741107.

15. Azevedo RT, De Beukelaer S, Jones IL, Safra L, Tsakiris M: When the lens is too wide: The political consequences of the visual dehumanization of refugees. Humanit Sco Sci Commun 2021, 8(1):1-16. https://doi.org/10.1057/s41599-021-00786-x. 
16. Passini S: Backing unequal policies: The complicit role of intergroup indifference. Peace and Conflict: J Peace Psychol 2019, 25(2):122. https://doi.org/10.1037/pac0000350.

17. Kotzur PF, Schäfer SJ, Wagner U: Meeting a nice asylum seeker: Intergroup contact changes stereotype content perceptions and associated emotional prejudices, and encourages solidarity-based collective action intentions. Br J Psychol 2019, 58(3):668-690. https://doi.org/10.1111/bjso.12304.

18. Vezzali L, Hewstone M, Capozza D, Trifiletti E, Bernardo GAD: Improving intergroup relations with extended contact among young children: Mediation by intergroup empathy and moderation by direct intergroup contact. $J$ Comm \& $\mathrm{Appl} S o \mathrm{C}$ Psychol 2017, 27(1):35-49. https://doi.org/10.1002/casp.2292.

19. Vezzali L, Stathi S, Crisp RJ, Giovannini D, Capozza D, Gaertner, SL: Imagined intergroup contact and common ingroup identity. Soc Psychol 2015, 46 (5):26576. https://doi.org/10.1027/1864-9335/a000242.

20. Verkuyten M: Emotional reactions to and support for immigrant policies: Attributed responsibilities to categories of asylum seekers. Soc Justice Res 2004, 17(3):293314. https://doi.org/10.1023/B:SORE.0000041295.83611.dc.

21. Verkuyten M, Mepham K, Kros M: Public attitudes towards support for migrants: the importance of perceived voluntary and involuntary migration. Ethn Racial Stud 2018, 41(5):901-918. https://doi.org/10.1080/01419870.2017.1367021.

22. Taylor LK, Glen C: From empathy to action: Can enhancing host-society children's empathy promote positive attitudes and prosocial behaviour toward refugees? $J$ Community Appl Soc Psychol 2020, 30:214-226. https://doi.org/10.1002/casp.2438.

23. Kotzur PF, Forsbach N, Wagner U: Choose your words wisely: Stereotypes, emotions, and action tendencies towards fled persons as a function of the group label. Soc Psychol 2017, 48(4): 226-241. https://doi.org/10.1027/1864-9335/a000312. 
24. Wyszynski MC, Guerra R, Bierwiaczonek K: Good refugees, bad migrants? Intergroup helping orientations toward refugees, migrants, and economic migrants in Germany. J Appl Soc Psychol 2020, 50(10):607-618. https://doi.org/10.1111/jasp.12699.

25. Pittinsky TL, Diamante N: Global bystander nonintervention. Peace and Conflict: $J$ Peace Psychol 2015, 21(2):226-247. https://doi.org/10.1037/pac0000076.

26. Goodman S, Kirkwood S: Political and media discourses about integrating refugees in the UK. Eur J Soc Psychol 2019, 49(7):1456-1470. https://doi.org/10.1002/ejsp.2595.

27. Atkinson M: Refugee mentoring: Sharing the journey. Peace and Conflict: J Peace Psychol 2018, 24(3):338-342. https://doi.org/10.1037/pac0000316.

28. Lamping S, Bertolo M, Wahlrab T: Activist citizens in an immigrant-friendly city: The Natural Helpers Program. Peace and Conflict: J Peace Psychol 2018, 24(3):330337. https://doi.org/10.1037/pac0000312.

29. Kip A, Priebe S, Holling H, Morina N: Psychological interventions for posttraumatic stress disorder and depression in refugees: A meta-analysis of randomized controlled trials. Clinical Psychol \& Psychoth 2020, 27(4):489-503. https://doi.org/10.1002/cpp.2446.

30. Schlechter P, Hellmann JH, Wingbermühle P, Morina N: Which psychological characteristics influence therapists' readiness to work with refugees? Clinical Psychol \& Psychoth 2021, 28(2):334-344. https://doi.org/10.1002/cpp.2508.

31. Steil R, Meichsner FL, Johow J, Krüger-Gottschalk A, Mewes R, Reese JP, Schumm H, Weise C, Morina N, Ehring T: Brief imagery rescripting vs. usual care and treatment advice in refugees with posttraumatic stress disorder: study protocol for a multi-center randomized-controlled trial. European Journal of Psychotraumatology 2021, 12(1):1872967. https://doi.org/10.1080/20008198.2021.1872967 
32. Schlechter P, Kamp S, Wanninger K, Knausenberger J, Wagner U, Wilkinson PO, Nohr L, Hellmann JH: Help-seeking attitudes and distress disclosure among Syrian refugees in Germany and German residents. The Counseling Psychologist 2021, 49(3):330-352. https://doi.org/10.1177/0011000020974934.

33. Schlechter P, Wilkinson PO, Knausenberger J, Wanninger K, Kamp S, Morina N, Hellmann JH: Depressive and anxiety symptoms in refugees: Insights from classical test theory, item response theory, and network analysis. Clinical Psychol \& Psychoth 2021, 28(1):169-181. https://doi.org/10.1002/cpp.2499.

34. Hellmann, JH, Forthmann, B, Knausenberger, J, Hellmann, DF, Rees, JH, Gansel, E, Back, MD, Echterhoff, G: Support for refugee integration in West and East Germany: Results from two lost letter studies. Soc Psychol 2020, 51(2):106115. https://doi.org/10.1027/1864-9335/a000397.

35. *Böhm R, Theelen MMP, Rusch H, Van Lange PAM: Costs, needs, and integration efforts shape helping behavior toward refugees. PNAS 2018, 115:7284-7289. https://doi.org/10.1073/pnas.1805601115.

36. *Glen C, Taylor LK, Dautel J: Promoting prosocial behavior toward refugees: Exploring the empathy-attitude-action model in middle childhood. In N. Bavlin, \& D. Christie (Eds.), Children and peace: From research to action. Springer Peace Psychology Book Series: Switzerland; 2019.

37. ${ }^{* *} \mathrm{Knab}$ N, \& Steffens MC: Emotions for solidarity: The relations of moral outrage and sympathy with hierarchy-challenging and prosocial hierarchy-maintaining action intentions in support of refugees. Peace and Conflict: J Peace Psychol 2021. Advance online publication. https://doi.org/10.1037/pac0000548.

Two studies examined predictors of prosocial action tendencies towards refugees and illustrate that moral outrage was more likely related to hierarchy-challenging actions 
such as solidarity-based collective action, whereas sympathy was more likely related to hierarchy-maintaining actions such as dependency-oriented helping.

38. *Thomas EF, Smith G, McGarty C, Reese G, Kende A, Bliuc A-M, Curtin N Spears R: When and how social movements mobilize action within and across nations to promote solidarity with refugees. Eur J Soc Psychol 2019, 49:213-229. https://doi.org/10.1002/ejsp.2380.

39. Siem B: Freiwilliges Engagement im Kontext von Flucht und Migration. [Volunteerism in the context of forced migration]. In A. Rohmann \& S. Stürmer (Eds.), Die Flüchtlingsdebatte in Deutschland - Sozialpsychologische Perspektiven: Beiträge zur Angewandten Psychologie [The refugee debate in Germany - social psychological perspectives: Essays for applied psychology] (pp. 29-52). Peter Lang; 2018.

40. Stürmer S, Siem B: A group-level theory of helping and altruism within and across group boundaries. In van E. Leeuwen \& H. Zagefka (Eds.), Intergroup helping (pp. 103-127). Springer; 2017.

41. *Louis WR, Thomas E, Chapman CM, Achia T, Wibisono S, Mirnajafi Z, Droogendyk L: Emerging research on intergroup prosociality: Group members' charitable giving, positive contact, allyship, and solidarity with others. Soc Personal Psychol Compass 2019; 13:e12436. https://doi.org/10.1111/spc3.12436.

42. Thomas EF, McGarty C: Giving versus acting: Using latent profile analysis to distinguish between benevolent and activist support for global poverty reduction. Br J Soc Psychol 2018, 57:189-209. https://doi.org/10.1111/bjso.12228

43. *Halabi S, Nadler A: The intergroup status as helping relations model: Giving, seeking and receiving help as tools to maintain or challenge social inequality. In: van Leeuwen E., Zagefka H. (eds) Intergroup Helping. Springer, Cham; 2017. https://doi.org/10.1007/978-3-319-53026-0_10. 
44. Halabi S, Dovidio JF, Nadler A: When intergroup helping helps intergroup relations: The moderating role of trust in the outgroup. J Exp Soc Psychol 2021, 95. https://doi.org/10.1016/j.jesp.2021.104141.

45. **Becker JC, Ksenofontov I, Siem B, Love A: Antecedents and Consequences of Autonomy- and Dependency-Oriented Help Toward Refugees. Eur J Soc Psychol 2019, 49:831-838. https://doi.org/10.1002/ejsp.2554.

This paper illustrates that paternalism is positively related to dependency-oriented help towards refugees, but negatively related to autonomy-oriented help. Both refugees and non-refugees perceived that autonomy-oriented help is more likely to bring about social change compared to dependency-oriented help.

46. Nadler A: Social psychology of helping relations: Solidarity and hierarchy. John Wiley \& Sons; 2020.

47. Lantos NA, Kende A, Becker JC, McGarty C: Pity for economically disadvantaged groups motivates donation and ally collective action intentions. Eur J Soc Psychol 2020, 50:1478-1499. https://doi.org/10.1002/ejsp.2705.

48. Schroeder J, Waytz A, Epley N: Endorsing help for others that you oppose for yourself: Mind perception alters the perceived effectiveness of paternalism. JEP: General 2017, 146(8):1106-1125. https://doi.org/10.1037/xge0000320.

49. Becker JC, Wright SC: Can cross-group contact turn advantaged group members into allies? The role of inequality-delegitimizing contact and interpersonal connection. Group Proc Intergroup Relat 2021. https://doi.org//10.1177/13684302211015273.

50. Kutlaca M, Radke HRM, Iyer A, Becker JC: Understanding allies' participation in social change: A multiple perspectives approach. Eur J Soc Psychol 2020, 50:12481258. doi: 10.1002/ejsp.2720.

51. Radke HRM, Kutlaca M, Siem B, Wright SC, Becker JC: Beyond allyship: Motivations for advantaged group members to engage in action for disadvantaged groups. 
Pers Soc Psychol Review 2020, 24(4):291-315.

https://doi.org/10.1177/1088868320918698.

52. Rosenberger S, Stern V, Merhaut N: Protest movements in asylum and deportation. Springer Nature; 2018.

53. Wibisono S, Mirnajafi Z, Droogendyk L, Chapman CM, Achia T, Thomas EF, Louis WR: Teaching and learning guide for intergroup prosociality. Soc Personal Psychol Compass 2019; 13:e12473. https://doi.org/10.1111/spc3.12473.

54. Alfadhli K, \& Drury, J: The role of shared social identity in mutual support among refugees of conflict: An ethnographic study of Syrian refugees in Jordan. J Comm Appl Soc Psychol 2018, 28(3):142-155. https://doi.org/10.1002/casp.2346.

55. Lyytinen E: Informal places of protection: Congolese refugees' 'communities of trust' in Kampala, Uganda. J Ethnic Migr Stud 2017, 43(6):991-1008. https://doi.org/10.1080/1369183X.2016.1207506.

56. Shaw SA: Bridge builders: A qualitative study exploring the experiences of former refugees working as caseworkers in the United States. J Soc Serv Res 2014, 40(3):284-296. https://doi.org/10.1080/01488376.2014.901276.

57. Weng SS, Lee JS: Why do immigrants and refugees give back to their communities and what can we learn from their civic engagement? VOLUNTAS: Intern J Vol Nonprof Organ 2015, 27:509-524. https//doi.org/10.1007/s11266-015-9636-5. 